\section{Prof. Hosten zum Geburtstag}

Am 14. Juli 2017 wird Prof. Dr. Norbert Hosten, Direktor des Instituts für Diagnostische Radiologie und Neuroradiologie der Universitätsmedizin Greifswald, 60 Jahre alt.

Prof. Hosten wurde in Düsseldorf geboren und studierte nach dem Abitur Humanmedizin an der Freien Universität Berlin, wo er 1987 promovierte. Er arbeitete am Rudolf Virchow Klinikum der Freien Universität Berlin in der Radiologie und Nuklearmedizin unter Prof. Dr. Dr. h. c. R. Felix zunächst als Assistenzarzt und zuletzt viele Jahre als Leitender Oberarzt. 1994 folgten die Habilitation und die Venia legendi im Fach Radiologie sowie 1999 die Berufung zur apl-Professur der Humboldt-Universität Berlin. Er erlangte 1999 die Schwerpunktbezeichnung Neuroradiologie und im Jahr 2000 den Facharzt für Nuklearmedizin.

Der klinische und wissenschaftliche Schwerpunkt von Prof. Hosten sind die lokal ablativen Verfahren zur Behandlung von Leber- und Lungentumoren, in dem er zusammen mit Prof. Thomas Vogl und Prof. Ulrich Speck Pionierarbeit leistete. Als Mitantragsteller und wissenschaftlicher Koordinator des Graduiertenkollegs 331 „Temperaturabhängige Effekte in Diagnostik und Therapie“ war Prof. Hosten die Schaltzentrale der verschiedenen Teilprojekte. Zu einer Zeit, in der die Bildnachverarbeitung noch weitgehend in der Hand von Informatikern lag, initiierte Prof. Hosten die Einrichtung eines Bildverarbeitungslabors, in dem beispielsweise die retrospektive Bildfusion von PET- und CToder MRT-Bildern zur Serienreife gebracht wurde.

Ein weiterer Schwerpunkt seiner Tätigkeit ist die Bildgebung der Orbita. Prof. Hosten erkannte früh das klinische und wissenschaftliche Potenzial der MRT dieser Region und motivierte seine Arbeitsgruppe zu experimentellen Arbeiten u. a. im Bereich von MRT-Oberflächenspulen und der Bestrahlungsplanung von Aderhautmelanomen.

Neben zahlreichen wissenschaftlichen Publikationen zu den genannten Themenbereichen hat er mehrere Monografien verfasst.

Prof. Hosten folgte 2001 dem Ruf auf den Lehrstuhl für Diagnostische Radiologie und Neuroradiologie der Universität Greifswald, wodurch nach 13 Jahren dieser Lehrstuhl wieder besetzt werden konnte. Er baute sein Institut sowohl innerhalb als auch außerhalb des Klinikums zu einer anerkannten Institution aus. So wurden unter seiner Leitung die Schwerpunkte Neuroradiologie und Kinderradiologie umfassend weiterentwickelt und auf den neuesten technischen Stand gebracht. Prof. Hosten hat die Radiologie stets als ein klinisches Fach begriffen. Daher ist ihm der konstruktive Dialog mit den klinischen Partnern sehr wichtig, sodass innerhalb des Universitätsklinikums sein Institut ein allseits anerkannter Gesprächspartner ist, dessen Rat sehr häufig gesucht wird. Wichtig sind ihm auch das Qualitätsmanagement und die stetige Verbesserung radiologischer Prozesse.

Seit 2008 ist Prof. Hosten Vorsitzender des Vereins „Telemedizin in der Euroregion POMERANIA e. V.“, dessen Ziel die grenzüberschreitende Verbessrung in der medizinischen Versorgung durch ein telemedizinisches Netzwerk ist. Der Verein erhielt 2012 den eGovernment Preis „Beste Lösung für ein gesellschaftliches Problem“. Prof. Hosten wurde 2015 für das von ihm geleitete Telemedizinprojekt in Szczecin mit dem Pomerania Nostra Preis ausgezeichnet.

Von 2013 bis 2015 war Prof. Hosten Präsident der Deutschen Röntgengesellschaft. Während dieser Zeit begleitete er die Entwicklung des e-Health-Gesetzes der Bundesregierung und setzte sich dabei für ein-

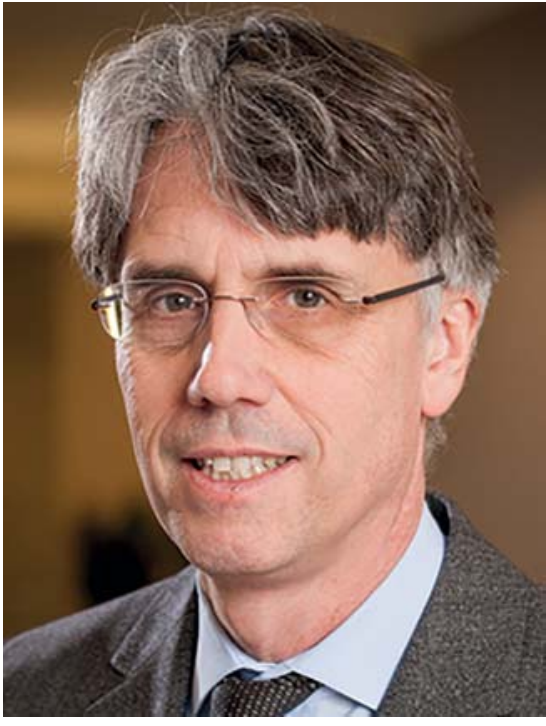

Prof. Dr. Norbert Hosten

heitliche Standards in der Teleradiologie ein. Die Ausstellung „Radiologie im Nationalsozialismus" wurde durch ihn in Israel präsentiert und mit seiner Unterstützung in das Hebräische übersetzt und in mehreren Städten Israels präsentiert, worauf eine enge Beziehung zu der Radiologie in Israel aufbaut. Basierend auf den Greifswalder Erfahrungen im Umgang mit „Big Data“ in der Radiologie führte Prof. Hosten während dieser Zeit eine DFG-Nachwuchsakademie durch und unterstütze maßgeblich die NaKo Gesundheitsstudie.

Die Atmosphäre in seinem Institut ist geprägt durch einen kooperativen Umgangsstil, wobei Wert auf sachlich fundierte Vorgehensweisen gelegt wird. Prof. Hosten vermag es, seine Mitarbeiter zu motivieren und anzuspornen. Darüber hinaus ist ihm die Förderung der wissenschaftlichen Tätigkeit seiner Mitarbeiter ein wichtiges Anliegen, wovon die hohe Qualität und das breite Spektrum der aus seinem Institut hervorgegangenen Arbeiten zeugen.

PD Dr. med. S. Langner, Greifswald PD Dr. med. B. Mensel, Greifswald Prof. Dr. med. A.-J. Lemke, Bremen PD Dr. med. M. Kirsch, Greifswald 